\title{
Integration of anatomical and external response mappings explains crossing effects in tactile localization: A probabilistic modeling approach
}

\author{
Stephanie Badde ${ }^{1,2} \cdot$ Tobias Heed $^{1} \cdot$ Brigitte Röder $^{1}$
}

Published online: 8 September 2015

(C) Psychonomic Society, Inc. 2015

\begin{abstract}
To act upon a tactile stimulus its original skinbased, anatomical spatial code has to be transformed into an external, posture-dependent reference frame, a process known as tactile remapping. When the limbs are crossed, anatomical and external location codes are in conflict, leading to a decline in tactile localization accuracy. It is unknown whether this impairment originates from the integration of the resulting external localization response with the original, anatomical one or from a failure of tactile remapping in crossed postures. We fitted probabilistic models based on these diverging accounts to the data from three tactile localization experiments. Hand crossing disturbed tactile left-right location choices in all experiments. Furthermore, the size of these crossing effects was modulated by stimulus configuration and task instructions. The best model accounted for these results by integration of the external response mapping with the original, anatomical one, while applying identical integration weights for uncrossed and crossed postures. Thus, the model explained the data without assuming failures of remapping. Moreover, performance differences across tasks were accounted for by non-individual parameter adjustments, indicating that individual participants' task adaptation results from one
\end{abstract}

Stephanie Badde

stephanie.badde@uni-hamburg.de

1 Biological Psychology and Neuropsychology, University of Hamburg, Von-Melle-Park 11, 20146 Hamburg, Germany

2 Department of Psychology, New York University, 6 Washington Place, 10003 New York, USA common functional mechanism. These results suggest that remapping is an automatic and accurate process, and that the observed localization impairments in touch result from a cognitively controlled integration process that combines anatomically and externally coded responses.

Keywords Tactile $\cdot$ Spatial localization $\cdot$ Reference frames $\cdot$ Mathematical models

\section{Introduction}

In touch, an object's location is initially encoded in a skinbased, anatomical reference frame: The spatial arrangement of neurons in the primary and secondary somatosensory cortex reflects the position of their receptive fields on the body surface (Penfield \& Boldrey, 1937). Yet, to plan actions toward the object, its location must be transformed into another, posture-dependent reference frame (Pouget et al., 2002; Sober \& Sabes, 2005). Any perceived tactile stimulus seems to be transformed into an external-spatial reference frame (Driver \& Spence, 1998; Yamamoto \& Kitazawa, 2001a; Shore et al., 2002; Spence et al., 2004; Soto-Faraco et al., 2004; Röder et al., 2004; Schicke \& Röder, 2006; Heed \& Azañón, 2014; Heed et al., 2015), even when such recoding is currently not required (Kitazawa, 2002; Azañón et al., 2010a). This process of coordinate transformation is addressed as tactile remapping (Driver \& Spence, 1998) and has been associated with regions of the intraparietal sulcus in posterior parietal cortex (Azañón et al., 2010b; Bolognini \& Maravita, 2007; Renzi et al., 2013). Crucially, both the original, anatomical representation as well as the remapped, external representation are maintained by the brain (Heed \& Röder, 2010; Buchholz et al., 2011; 2013), 
that is, after transformation both reference frames are available to estimate the location of the touch. Consequently, the brain might base tactile localization by default on information coded in both reference frames, rather than relying responses on the external reference frame alone.

To differentiate between different tactile reference frames, researchers have manipulated body posture to create situations in which anatomical and external reference frames point to conflicting left-right responses. For example, when the hands are crossed, the right hand (anatomical reference frame) is located in the left hemispace (external reference frame). A well-known consequence of hand crossing is that participants' ability to judge which of two successive tactile stimuli, one applied to each hand, occurred first is markedly impaired compared to an uncrossed hands condition (Yamamoto \& Kitazawa, 2001a; Shore et al., 2002). These so-called crossing effects in temporal order judgments (TOJ) are thought to arise from conflicting leftright mappings in the different spatial reference frames (Yamamoto \& Kitazawa, 2001a; 2001b; Shore et al., 2002; Röder et al., 2004; Schicke \& Röder, 2006; Heed et al., 2012; Heed \& Azañón, 2014; Azañón et al., 2015). However, it is unclear whether this conflict bears on the remapping process, impairing the establishment of the external response mapping (Yamamoto \& Kitazawa, 2001a; Röder et al., 2004; Kóbor et al., 2006; Azañón \& Soto-Faraco, 2007; Kitazawa et al., 2008), or whether it unfolds its effect at the stage of information integration once remapping is complete (Shore et al., 2002; Badde et al., 2013; 2014; Badde et al., 2015). Additionally, it has been suggested that crossing effects might simply be caused by the additional demands of the unusual, presumably uncomfortable crossed posture (Azañón et al., 2010b; Longo et al., 2010; Haggard et al., 2003) or by additional hemispheric transmission costs, which are specific to the crossed posture (Buchholz et al., 2012; Canzoneri et al., 2014). Furthermore, it has been debated whether crossing effects critically depend on the application of more than one stimulus in the TOJ task (Yamamoto \& Kitazawa, 2001a; Kitazawa, 2002; Shore et al., 2002). To address these questions, we used a two-step strategy. First, we tested whether tactile TOJ crossing effects are task- and stimulation-specific. To this end, we modified the original TOJ paradigm, by varying, within participants, the task instructions and the number of stimuli. Second, we compared the ability of different models to account for participants' behavior in these different tasks. These models were designed on the two theoretical accounts for crossing effects, that is, non-reliable externally coded response mapping in the crossed posture versus integration of conflicting left-right mappings in the crossed posture.

\section{Materials and methods}

\section{Behavioral experiments}

Participants Nineteen right-handed participants (six male, aged 20-37 years, mean 25 years) from the community of Hamburg volunteered for the experiment. All had normal or corrected-to-normal vision and did not report any impairments in tactile sensitivity. Participants received course credit or were compensated with 7 Euro/hour. The experiment was conducted in accordance with the general guidelines of the Declaration of Helsinki (World Medical Association, 2008).

Apparatus Participants were seated at a table, resting their hands and elbows on the table surface. The index fingers were placed on response buttons. The arms were positioned either crossed or uncrossed. A foam cushion was placed underneath one arm to avoid skin contact between the hands and arms in the crossed posture. The distance between the response buttons for the two hands was $25 \mathrm{~cm}$. Tactile stimulators (Oticon bone conductors, type BC 461-012, sized about $1.6 \times 1 \times 0.8 \mathrm{~cm}$ ) were taped to the middle fingers, covering the whole fingernail and some proximate skin. Stimuli consisted of 15-ms-long vibrations at a frequency of $200 \mathrm{~Hz}$. The experiment was controlled by the software Presentation, version 14.5 (Neurobehavioral Systems, Albany, CA, USA), which interfaced with custom-built hardware to drive the stimulators and to record responses. To shield off any auditory cues produced by the tactile stimulators, participants wore ear plugs as well as headphones that played white noise. Instructions and a fixation cross were displayed on a monitor placed $80 \mathrm{~cm}$ in front of the participant.

\section{Tasks}

Temporal order judgment (TOJ) Two tactile stimuli were successively presented, one stimulus to each hand. Participants were instructed to report the temporal order of the two stimuli and to respond by a button press with the hand that was stimulated first (Fig. 1).

First touch localization (FTL) Two tactile stimuli were successively presented, one stimulus to each hand. Participants were instructed to localize the first stimulus as fast as possible and to ignore the second stimulus. Responses were given by button press with the hand at which participants had perceived the relevant, first touch. Thus, the FTL task differed from the TOJ task only with respect to the task instructions. 


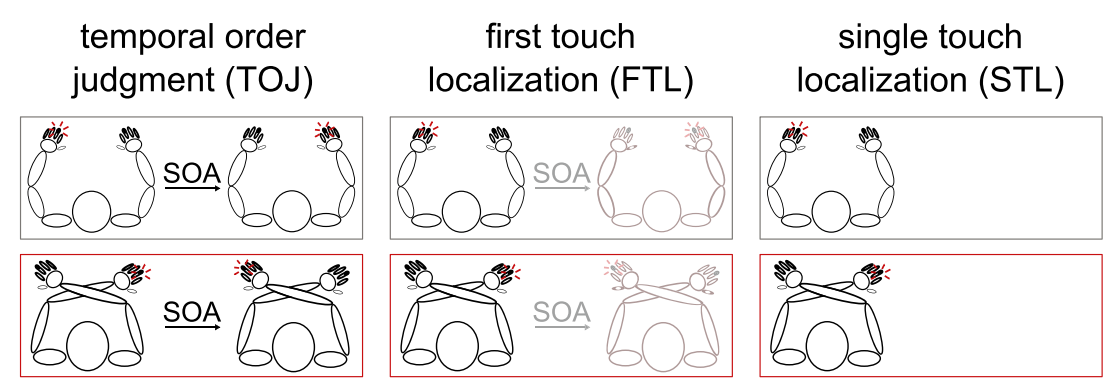

Fig. 1 The three touch localization tasks. In the temporal order judgment (TOJ) task and in the first touch localization (FTL) task two tactile stimuli were successively applied, one to each hand. In the TOJ task, participants were asked to determine the order of the two stimuli and to indicate the location of the first touch, whereas in the FTL

Single touch localization (STL) A single tactile stimulus was applied, either to the right or to the left hand. Participants had to localize the stimulus and respond by a button press with the respective hand. Thus, the STL task differed from the TOJ and FTL tasks in the number of presented stimuli. Instructions for all three localization tasks, TOJ, FTL, and STL, stressed response accuracy but not response speed.

Detection task One tactile stimulus was applied, either to the right or to the left hand. The task was to detect the stimulus and to respond as fast as possible with the designated response hand. The response hand was fixed throughout experimental blocks and was not related to the stimulated hand.

Design Two within-participant factors were manipulated in all experiments: hand posture (factor: posture, levels: uncrossed and crossed) and the hand which received the relevant (first) stimulus (factor: hand, levels: left and right hand). In the TOJ and FTL tasks, the time interval between the two stimuli was varied in addition (factor: stimulus onset asynchrony (SOA), levels: 50, 80, 110, 150, 200, 250, and $300 \mathrm{~ms}$ ). In the detection task, the stimulus either occurred at the designated response hand or at the other hand (factor: congruency, levels: congruent and incongruent). The response hand was either the left or the right hand (factor: response hand).

Procedure Every trial lasted 3000 ms. Each experiment comprised 200 trials, divided into four blocks of 50 trials each. Participants were encouraged to rest between blocks. The factors hand and SOA varied within blocks, whereas posture varied every other block. Condition order and the order of experiments were counterbalanced across participants. task participants had to ignore the second touch and to localize the first touch as fast as possible. In the single-touch localization task (STL) only one touch was applied either to the right or to the left hand and had to be localized. Additionally, participants performed a touch detection task, that is not depicted here

Data preparation Trials with reaction times below $150 \mathrm{~ms}$ and above $1500 \mathrm{~ms}$ were excluded from the analysis (3.5\% of all trials).

TOJ and FTL performance were quantified in two ways: first, by mean accuracy averaged across all SOAs (Cadieux et al., 2010), and second, by slope values of a probit regression over SOAs up to $250 \mathrm{~ms}$ (with negative SOA indicating "left hand first" stimuli; Fig. 2) (Shore et al., 2002). For the probit analysis (Finney, 1947), response accuracy was transformed into "right hand first" responses, indicating whether participants judged the anatomically right hand to be stimulated first (Yamamoto \& Kitazawa, 2001a; Shore et al., 2002). Then, probit values of the ratio of "right hand first" responses per SOA were calculated by applying the inverse normal distribution $\left(\operatorname{probit}\left(p_{\text {right hand first }}\right)=\right.$ $z \Leftrightarrow \Phi_{0,1}(z)=p_{\text {right hand first }) \text {. The resulting probit values }}$ were linearly regressed onto their corresponding SOA. The regression slope was used as a measure of TOJ performance with steeper probit slopes indicating better performance (Shore et al., 2002; Heed \& Azañón, 2014).

STL performance was quantified by mean accuracy only, as this task involved just one touch.

In the detection task, participants rarely missed a stimulus. Therefore, reaction time (RT) rather than accuracy was analyzed.

Data analysis A repeated measures ANOVA with factors posture and experiment was conducted on accuracy data from the three localization experiments (TOJ, FTL, and STL). Paired $t$ tests on mean accuracy and, where applicable, probit slopes were used to assess crossing effects separately for each experiment. Difference scores between uncrossed and crossed postures were calculated for accuracy and, where applicable, probit slopes. First, these difference scores were compared across experiments using pairwise paired $t$ tests. Second, for each pair of experiments Pearson's $r$ of the difference scores was calculated and tested for significant deviation from zero. 

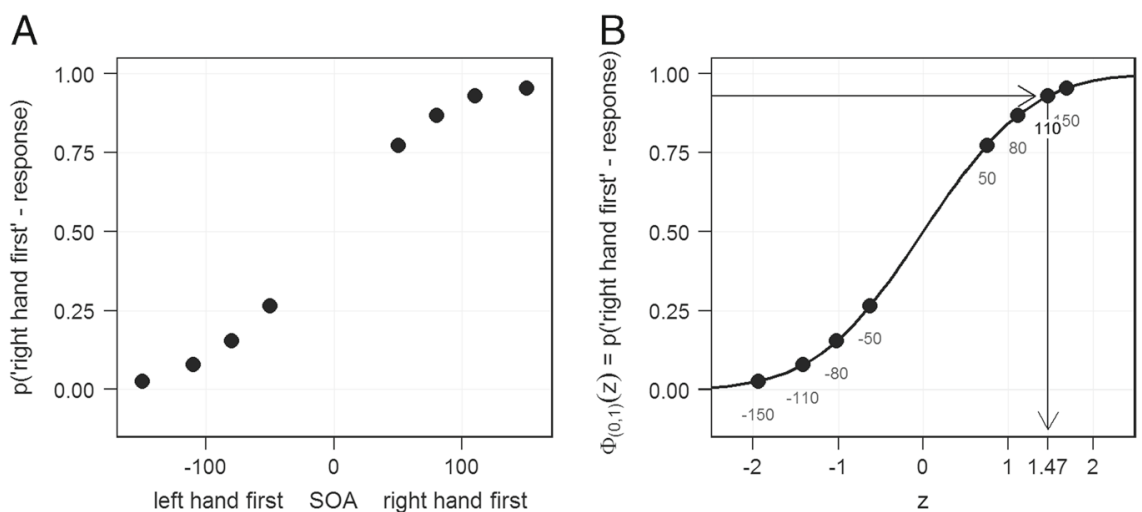

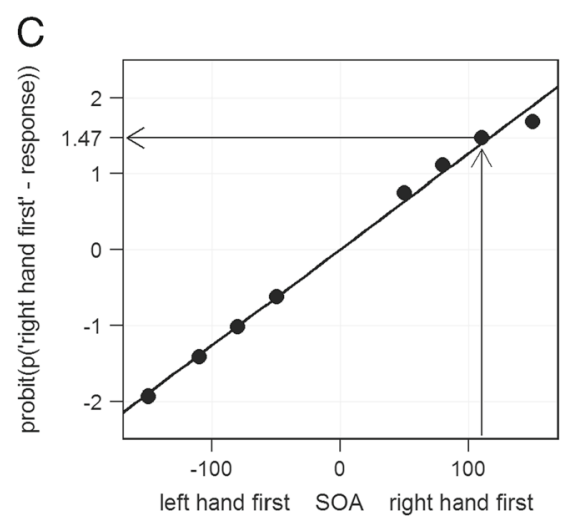

Fig. 2 Probit analysis of TOJ data. A Responses were transformed into 'right hand first' responses. The proportion of 'right hand first' responses was determined as a function of SOA with negative SOA indicating 'left hand first' stimuli. B Probit values for each proportion of 'right hand first' responses were calculated by applying the

For the detection task, a repeated measures ANOVA (type III sum of squares) with factors posture, response hand, and congruency was conducted on RT.

p-values were adjusted for multiple comparisons according to Holm (1979). All reported results were significant at a type I error level of $5 \%$ unless noted otherwise. $\eta^{2}$ and $d$ were used as standardized effect size measures. Note that $d$ was calculated from the mean difference and the standard deviation of the differences and, thus, is not directly comparable to $d$ values obtained in between subjects designs.

\section{Model structure}

We compared several variants of two main models. We termed the first main model integration model: this model explained crossing effects as resulting from integration of potentially conflicting anatomical and external left-right response mappings. We termed the second main model nonintegration model: this model explained crossing effects as resulting from reduced reliability of the external response mapping in the crossed posture.

Integration models Our integration model incorporates the idea that the probability of a right-hand response $\left(\operatorname{logit}^{-1}(\theta)\right)^{1}$ is based on the weighted combination of the response codes $(\rho)$ derived from anatomical and external reference frames.

$\theta=\omega_{\text {anatomical }} \rho_{\text {anatomical }}+\omega_{\text {external }} \rho_{\text {external }}^{1}$

\footnotetext{
$\left.\overline{{ }^{1} \theta \in(-\infty}, \infty\right)$ (logit of the probability of a right hand response); $\omega_{\text {anatomical }}, \omega_{\text {external }} \in[0, \infty)$ (weights); $\rho_{\text {anatomical }}, \rho_{\text {external }} \in$ $\{-1,1\}$ (left-right response codes); $\delta_{\text {anatomical }}, \delta_{\text {external }}$ (task context)
}

inverse normal distribution $\left(\operatorname{probit}\left(p_{\text {right hand first }}\right)=z \Leftrightarrow \Phi_{0,1}(z)=\right.$ $\left.p_{\text {right hand first }}\right)$. C The resulting probit values were linearly regressed onto their corresponding SOA. The regression slope was used as a measure of TOJ performance with steeper probit slopes indicating better performance

In the experiments that we report in the present study, tactile stimuli were always applied to one of two locations, the left hand or the right hand, with each hand positioned in the left or right hemispace. Thus, for both reference frames, the task-relevant spatial information could be reduced to leftright categories. We arbitrarily used -1 to code for left and 1 to code for right. The left-right mapping of the tactile stimuli depended not only on the stimulated hand, but in addition on hand posture as well. The anatomical mapping was constant across hand postures, whereas the external left-right mapping switched in the crossed posture: For example, the left crossed hand was located in right space, and, therefore, its external response code ( $\left.\rho_{\text {external }}\right)$ was 1 rather than -1 . As a consequence, anatomical and external response mappings were congruent in the uncrossed posture, so that the combination of the anatomical and external response codes $(\theta)$ was determined by the sum of their weights. In contrast, anatomical and external left-right mappings were opposed in the crossed posture, so that $\theta$ depended on the difference of their weights.

Responses were always coded relative to the right hand, as is custom in the analysis of TOJ crossing effects (Yamamoto and Kitazawa, 2001a; Shore et al., 2002). To allow comparisons across all three tasks, we initially averaged across SOAs in the TOJ and FTL tasks. Thus, the data consisted of the number of "right-hand" responses per participant, stimulated hand, and posture for each experiment. For each condition, the number of "right-hand" responses was described as a binomially distributed random variable. The inverse logit of $\theta$, that is, the inverse logit of the combined anatomical and external left-right response codes corresponded to the Bernoulli parameter of the Binomial, describing the probability to give a "right-hand" response in a single trial. The graphical model of one integration model variant is depicted in Fig. 3. 


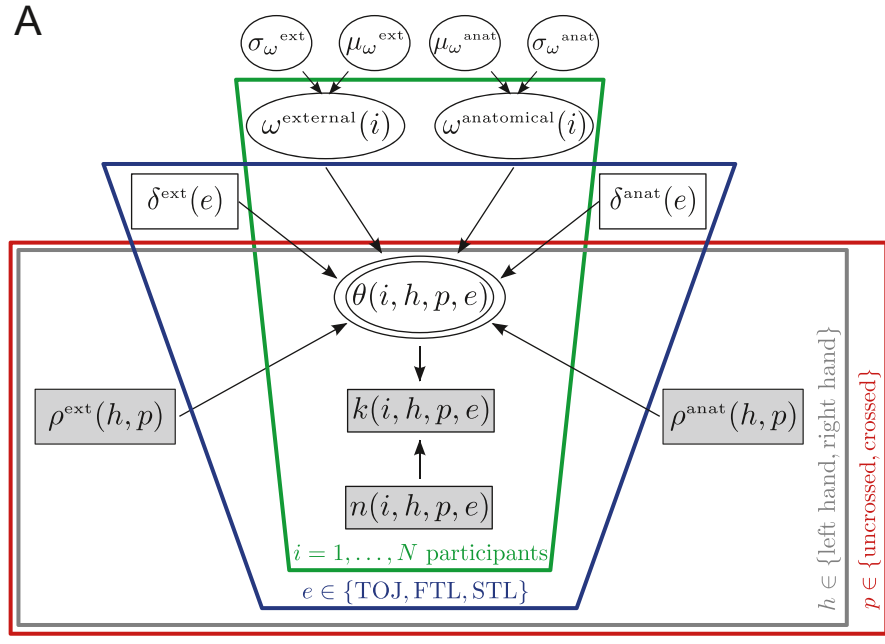

B

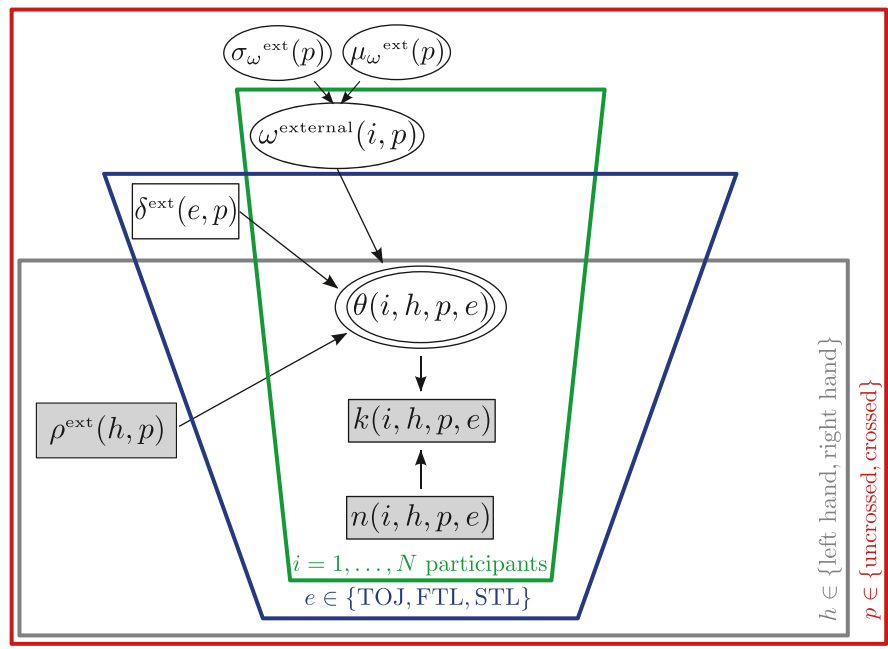

Fig. 3 Graphical description of the best integration model (A) and the according non-integration model (B). The probability to localize a touch to the right hand in a single trial $\left(\operatorname{logit}^{-1}(\theta)\right)$ was either derived from the stimulus's weighted anatomical and external leftright response codes ( $\rho^{\text {anat }}$ and $\left.\rho^{\text {ext }}\right)$ (integration model, A) or from its external response codes alone (non-integration model, B). For each participant $(i)$, the weight parameters $\left(\omega^{\text {anat }}\right.$ and $\left.\omega^{\text {ext }}\right)$ were drawn from a population distribution $\left(\mathcal{N}\left(\mu_{\omega}^{\text {anat }}, \sigma_{\omega}^{\text {anat }}\right)\right.$ and $\left.\mathcal{N}\left(\mu_{\omega}^{\text {ext }}, \sigma_{\omega}^{\text {ext }}\right)\right)$ which parameters were concurrently estimated by the model. No upper bound was set for the weights (an equivalent formulation of the model, that

$$
\begin{aligned}
& \theta(i, h, p, e)=\omega^{\text {anat }}(i) \rho^{\text {anat }}(h, p) \delta^{\text {anat }}(e)+\omega^{\text {ext }}(i) \rho^{\text {ext }}(h, p) \delta^{\text {ext }}(e) \\
& k(i, h, p, e) \sim \operatorname{Binomial}\left(\text { logit }^{-1}(\theta(i, h, p, e)), n(i, h, p, e)\right) \\
& \delta^{\text {anat }}(e) \sim \mathcal{N}(1,2) \\
& \delta^{\text {ext }}(e) \sim \mathcal{N}(1,2) \\
& \omega^{\text {anat }}(i) \sim \mathcal{N}\left(\mu_{\omega}{ }^{\text {anat }}, \sigma_{\omega}{ }^{\text {anat }}\right) I_{[0, \infty)} \\
& \omega^{\text {ext }}(i) \sim \mathcal{N}\left(\mu_{\omega}{ }^{\text {ext }}, \sigma_{\omega}{ }^{\text {ext }}\right) I_{[0, \infty)} \\
& \mu_{\omega}{ }^{\text {anat }} \sim \mathcal{N}(1,2) \quad \sigma_{\omega}{ }^{\text {anat }} \sim \text { Uniform }(0,2) \\
& \mu_{\omega}^{\text {ext }} \sim \mathcal{N}(1,2) \quad \sigma_{\omega}{ }^{\text {ext }} \sim \text { Uniform }(0,2) \\
& k(i, h, p, e) \in\left\{0, \ldots, n_{i j}\right\} \quad \rho^{\text {anat }}(h, p) \in\{-1,1\} \\
& n(i, h, p, e) \in\{0, \ldots, 100\} \quad \rho^{\text {ext }}(h, p) \in\{-1,1\}
\end{aligned}
$$

$$
\begin{aligned}
& \theta(i, h, p, e)=\omega^{\text {ext }}(i, p) \rho^{\text {ext }}(h, p) \delta^{\text {ext }}(p, e) \\
& k(i, h, p, e) \sim \operatorname{Binomial}\left(l_{\text {ogit }}{ }^{-1}(\theta(i, h, p, e)), n(i, h, p, e)\right) \\
& \delta^{\text {ext }}(p, e) \sim \mathcal{N}(1,2) \\
& \omega^{\text {ext }}(i, p) \sim \mathcal{N}\left(\mu_{\omega}{ }^{\text {ext }}, \sigma_{\omega}{ }^{\text {ext }}\right) I_{[0, \infty)} \\
& \mu_{\omega}{ }^{\text {ext }}(e) \sim \mathcal{N}(1,2) \quad \sigma_{\omega}{ }^{\text {ext }}(e) \sim \operatorname{Uniform}(0,2) \\
& \rho^{\text {ext }}(h, p) \in\{-1,1\} \\
& n(i, h, p, e) \in\{0, \ldots, 100\} \\
& k(i, h, p, e) \in\left\{0, \ldots, n_{i j}\right\}
\end{aligned}
$$

incorporates bound weights summing to one is presented in the Supplementary Material). In both models the individual weights were adjusted to the three different tasks (see Fig. 1) by non-individual task context parameters ( $\delta^{\text {anat }}$ and $\delta^{\text {ext }}$ ). In both models weights varied across individuals (green frame), but the task context parameters did not (blue frame). Crucially, in the integration model (A) none of the free parameters (non-shaded boxes) varied across postures (red frame), whereas all free parameters were estimated separately for uncrossed and crossed postures in the non-integration model (B, red frame)

an independent estimate of the inflection point of the sigmoid, the point of subjective simultaneity (PSS) $\left(\theta^{\prime}(s)=\right.$ $\theta \cdot \mathrm{SOA}(s) /$ const $+\mathrm{PSS})$.

We tested several variants of this integration model to explore the consistency of the incorporated reference frames weights $\left(\omega_{\text {anatomical }}\right.$ and $\left.\omega_{\text {external }}\right)$ across experiments, postures, and participants. In model terms, we gradually reduced the number of free parameters based on theoretical assumptions. Model variants are listed in Table 1. 
Table 1 Goodness of fit of all tested models

\begin{tabular}{|c|c|c|c|c|c|c|}
\hline & $R^{2}$ & RMSD & B. $p$ & BPIC & $\# 1$ & \# 2 \\
\hline \multicolumn{7}{|l|}{ Integration model variants - binomial (SOA-independent) models } \\
\hline $1 \theta(i, h, p, e)=\omega_{\text {anat }}(i, p, e) \rho_{\text {anat }}(h, p)+\omega_{\mathrm{ext}}(i, p, e) \rho_{\mathrm{ext}}(h, p)$ & 0.97 & 5.59 & 0.52 & 1361 & 12 & 12 \\
\hline $2 \theta(i, h, p, e)=\omega_{\text {anat }}(i, p) \rho_{\text {anat }}(h, p) \delta_{\text {anat }}(p, e)+\omega_{\mathrm{ext}}(i, p) \rho_{\mathrm{ext}}(h, p) \delta_{\mathrm{ext}}(p, e)$ & 0.96 & 5.87 & 0.52 & 1369 & 4 & $4+4$ \\
\hline $3 \theta(i, h, p, e)=\omega_{\text {anat }}(i) \rho_{\text {anat }}(h, p) \delta_{\text {anat }}(e)+\omega_{\text {ext }}(i) \rho_{\text {ext }}(h, p) \delta_{\text {ext }}(e)$ & 0.96 & 6.03 & 0.51 & 1324 & 2 & $2+2$ \\
\hline $4 \theta(i, h, p, e)=\omega_{\text {anat }} \rho_{\text {anat }}(h, p) \delta_{\text {anat }}(e)+\omega_{\text {ext }}(i) \rho_{\text {ext }}(h, p) \delta_{\text {ext }}(e)$ & 0.94 & 7.04 & 0.47 & 1507 & 1 & $1+2$ \\
\hline $5 \theta(i, h, p, e)=\omega_{\text {anat }}(i) \rho_{\text {anat }}(h, p) \delta_{\text {anat }}(e)+\omega_{\text {ext }} \rho_{\text {ext }}(h, p) \delta_{\text {ext }}(e)$ & 0.94 & 7.52 & 0.45 & 1762 & 1 & $1+2$ \\
\hline $6 \theta(i, h, p, e)=\pi(i)\left(\omega_{\text {anat }} \rho_{\text {anat }}(h, p) \delta_{\text {anat }}(e)+\omega_{\text {ext }} \rho_{\text {ext }}(h, p) \delta_{\text {ext }}(e)\right)$ & - & - & - & - & 1 & $1+2$ \\
\hline \multicolumn{7}{|l|}{ Integration model variants - logistic (SOA-dependent) models } \\
\hline $7 \theta^{\prime}(i, h, p, e)=\left(\omega_{\text {anat }}(i) \rho_{\text {anat }}(p) \delta_{\text {anat }}(e)+\omega_{\text {ext }}(i) \rho_{\text {ext }}(p) \delta_{\text {ext }}(e)\right) \cdot \operatorname{SOA}(s) /$ const & 0.78 & 8.77 & 0.34 & 10867 & 2 & $2+2$ \\
\hline $8 \theta^{\prime}(i, h, p, e)=\left(\omega_{\mathrm{anat}}(i) \rho_{\mathrm{anat}}(p) \delta_{\mathrm{anat}}(e)+\omega_{\mathrm{ext}}(i) \rho_{\mathrm{ext}}(p) \delta_{\mathrm{ext}}(e)\right) \cdot \operatorname{SOA}(s) /$ const $+\operatorname{PSS}(i)$ & 0.80 & 8.51 & 0.39 & 10418 & 3 & $2+2$ \\
\hline \multicolumn{7}{|l|}{ Non-integration model variants - binomial (SOA-independent) models } \\
\hline $9 \theta(i, h, p, e)=\omega_{\mathrm{ext}}(i, h, p, e) \rho_{\mathrm{ext}}(h, p)$ & 0.98 & 3.97 & 0.61 & 1334 & 12 & 12 \\
\hline $10 \theta(i, h, p, e)=\omega_{\mathrm{ext}}(i, p, e) \rho_{\mathrm{ext}}(h, p)$ & 0.97 & 5.59 & 0.51 & 1398 & 6 & 6 \\
\hline $11 \theta(i, h, p, e)=\omega_{\mathrm{ext}}(i, p) \rho_{e x t}(h, p) \delta_{\mathrm{ext}}(p, e)$ & 0.91 & 8.95 & 0.44 & 1972 & 2 & $2+2$ \\
\hline \multicolumn{7}{|l|}{ Non-integration model variants - logistic (SOA-dependent) models } \\
\hline $12 \theta^{\prime}(i, p, e, s)=\omega_{\mathrm{ext}}(i, p, e) \rho_{\mathrm{ext}}(p) \cdot \operatorname{SOA}(s) /$ const $+\operatorname{PSS}(i, p, e)$ & 0.84 & 7.63 & 0.39 & 9172 & 24 & 12 \\
\hline $13 \theta^{\prime}(i, p, e, s)=\omega_{\mathrm{ext}}(i, p) \rho_{\mathrm{ext}}(p) \delta_{\mathrm{ext}}(p, e) \cdot \operatorname{SOA}(s)+\operatorname{PSS}(i)$ & 0.78 & 8.75 & 0.34 & 10970 & 3 & $2+2$ \\
\hline
\end{tabular}

B. $\mathrm{p}$ refers to the Bayesian $p$ value, \# 1 contains the number of parameters per participant, and \# 2 contains the number of population level parameters

First, we tested a model that only had the constraint that stimulus localization to the left and to the right hand $(h)^{2}$ was described with the same set of weight parameters, but allowed for separate anatomical and external weight parameter estimates for each individual $(i)$, posture $(p)$, and experiment $(e)$ (Table 1, Model 1):

Model1: $\begin{aligned} \theta(i, h, p, e)= & \omega_{\text {anatomical }}(i, p, e) \rho_{\text {anatomical }}(h, p) \\ & +\omega_{\text {external }}(i, p, e) \rho_{\text {external }}(h, p)^{1,2}\end{aligned}$

Yet, our experiments were based on the hypothesis that crossing effects are caused by conflicting anatomical and external left-right mappings in the crossed posture, implying that crossing effects are related in all three localization experiments. Consequently, we devised a model that described the data from all three localization experiments simultaneously by introducing one task context parameter for each reference frame $\left(\delta_{\text {anatomical }}\right.$ and $\left.\delta_{\text {external }}\right)$. These task context parameters adjusted the individual anatomical and external weights across all participants to capture general differences between responses in the three experiments. The anatomical and external task contexts ( $\delta_{\text {anatomical }}$ and

$\overline{2_{i}=1, \ldots, n}$ (participant), $h \in\{$ left, right $\}$ (stimulated hand), $p \in$ $\{$ uncrossed, crossed $\}$ (posture), $e \in\{$ TOJ, FTL, STL $\}$ (experiment) $\left.\delta_{\text {external }}\right)$ of the TOJ task served as reference for the other two tasks and were, accordingly, set to 1 . Task context parameters for the STL and FTL tasks were allowed to vary across postures. Thus, in this model variant the individual reference frame weights ( $\omega_{\text {anatomical }}$ and $\left.\omega_{\text {external }}\right)$ still varied across postures but were now constant across experiments (Table 1, Model 2):

Model 2: $\theta(i, h, p, e)=\omega_{\text {anatomical }}(i, p) \rho_{\text {anatomical }}(h, p) \delta_{\text {anatomical }}(p, e)$ $+\omega_{\text {external }}(i, p) \rho_{\text {external }}(h, p) \delta_{\text {external }}(p, e)^{1,2}$

In the next model variant, we tested whether participants changed their reference frame weighing with posture, by restricting the participant-specific weights ( $\omega_{\text {anatomical }}$ and $\left.\omega_{\text {external }}\right)$ to be constant across postures. We term this model "pure conflict model", because it attempts to explain crossing effects only by individually weighted anatomical and external left-right response codes. Accordingly, the task context parameters ( $\delta_{\text {anatomical }}$ and $\left.\delta_{\text {external }}\right)$ were also held constant across postures (Table 1, Fig. 3, Model 3):

Model 3: $\theta(i, h, p, e)=\omega_{\text {anatomical }}(i) \rho_{\text {anatomical }}(h, p) \delta_{\text {anatomical }}(e)$ $+\omega_{\text {external }}(i) \rho_{\text {external }}(h, p) \delta_{\text {external }}(e)^{1,2}$

To further reduce the number of free parameters, we next tested whether it is necessary to assume that both anatomical and external weights vary across participants. To address 
this question, either the anatomical or the external reference frame weights ( $\omega_{\text {anatomical }}$ and $\left.\omega_{\text {external }}\right)$ were held constant across participants (Table 1, Models 4 and 5):

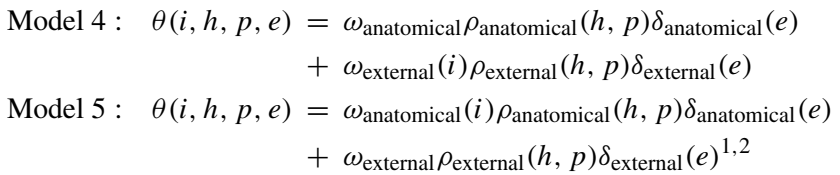

Next, we tested an integration model variant with anatomical and external reference frame weights held constant across participants. This model explained interindividual differences only by a general performance level parameter $(\pi)$ (Table 1, Model 6): Model 6: $\theta(i, h, p, e)=\pi(i) \cdot\left(\omega_{\text {anatomical }} \rho_{\text {anatomical }}(h, p) \delta_{\text {anatomical }}(e)\right.$
$\left.+\omega_{\text {external }} \rho_{\text {external }}(h, p) \delta_{\text {external }}(e)\right)^{1,2}$

We designed two logistic models to assess the effects of SOA and fitted them to the data from TOJ and FTL tasks. In addition, these models tested whether participants had an individual bias towards one of the hands by including a free PSS parameter (Table 1, Models 7 vs. 8): ${ }^{3}$

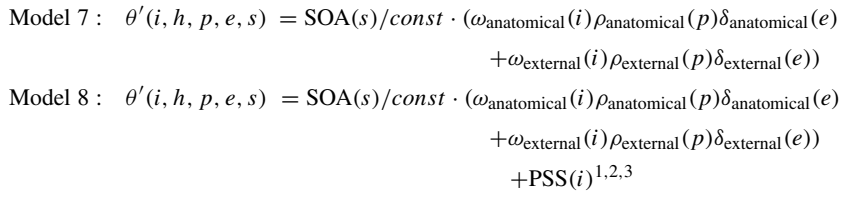

Non-integration models The second set of models were based on the hypothesis that crossing effects in tactile localization tasks result from unreliable external left-right mappings in the crossed but not in the uncrossed posture. This assumption was modeled by varying the weight given to the correctly mapped external response code between crossed and uncrossed postures. In contrast to the integration models, the non-integration models comprised the external, but not the anatomical left-right response code. Consequently, only one weight rather than two weights were estimated to calculate the logit of the probability of a right-hand response $(\theta)$. This design reflects that integration of the transformed

\footnotetext{
${ }^{3} s \in\{-300,-250,-200,-150,-110,-80-50,50,80,110,150$, $200,250,300\}$ (SOA, with negative SOA indicating stimuli to the left hand). As SOA coded for the hand, which received the first touch, $\rho$ in the logistic models only depends on posture $(p)$ and not on the stimulated hand. For simplicity, the scaling constant const was set to 200 in the final model. The calculation of $\theta^{\prime}$ was based on $\theta$ as calculated in Model 3.
}

external with the original, skin-based response codes is not assumed within this account. Again, the number of free parameters was reduced over a series of model variants.

We started with a binomial model without any constraints. That is, separate response probabilities, $\operatorname{logit}^{-1}(\theta)$, (Model 9) were calculated for each participant, stimulated hand, posture, and experiment. As in the integration model, $\operatorname{logit}^{-1}(\theta)$ was implemented as a Bernoulli parameter. This model had no explanatory value, as one parameter per data point was fitted, but rather provided a measure of the goodness of fit that could be maximally achieved by fitting a binomial model to our data (Table 1, Model 9):

Model 9: $\theta(i, h, p, e)=\omega_{\text {external }}(i, h, p, e) \rho_{\text {external }}(h, p)^{1,2}$

First, we constrained the non-integration model to describe the localization of stimuli on both hands with the same set of weights, but allowing the external reference frame weight to differ across participants, postures, and experiments (Table 1, Model 10):

Model 10: $\theta(i, h, p, e)=\omega_{\text {external }}(i, p, e) \rho_{\text {external }}(h, p)^{1,2}$

Second, we constrained the non-integration model to explain data from all three experiments simultaneously (Table 1, Model 11), allowing individual weights for each posture, but using these parameters for all three experiments. Differences between experiments were again captured by a task context parameter $\left(\delta_{\text {external }}\right)$ that varied across experiments and postures. The number of parameters in this non-integration model variant was equal to the number of parameters of the pure conflict model (Model 3, Fig. 3).

Model 11: $\quad \theta(i, h, p, e)=\omega_{\text {external }}(i, p) \rho_{\text {external }}(h, p) \delta_{\text {external }}(p, e)^{1,2}$

In analogy to the integration model, we extended the nonintegration model by implementing the factor SOA for the TOJ and FTL tasks. In these logistic models (Models 12 and 13), the weighted external left-right response $\operatorname{code} \theta$ was used as the slope of a logistic function and, additionally, a separate PSS parameter was estimated for each sigmoid. Again, we started with an unconstrained model, estimating separate parameters for each participant, stimulated hand, posture, and experiment, to show the maximal fit achievable with a logistic model (Table 1, Model 12).

Model 12: $\quad \theta^{\prime}(i, p, e, s)=\omega(i, p, e) \rho(p) \cdot \operatorname{SOA}(s) /$ const $+\operatorname{PSS}(i, p, e)^{1,2,3}$

Finally, we constructed a logistic variant of the nonintegration model that allowed the external weight to vary 
across participants and postures, but not across experiments (Table 1, Model 12). This model had the same number of free parameters as the corresponding integration model, Model 8.

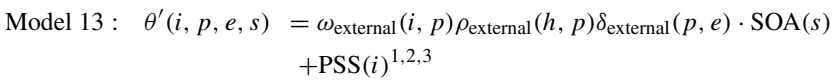

Model implementation The posterior distributions of each model parameter were approximated with Markov Chain Monte Carlo (MCMC) sampling using WinBUGS (Lunn et al., 2000). Three Markov chains of 1000 samples were initialized with random values. The first 100 samples were disregarded to allow the chains to reach their equilibrium distribution (Rouder \& Lu, 2005).

We employed a hierarchical model design that allowed predicting response probabilities of new participants. Thus, free parameters that varied across participants were drawn from an overarching population distribution, rather than being estimated independently. The only free parameters that varied across participants were the anatomical and external weight parameters ( $\omega_{\text {anatomical }}$ and $\left.\omega_{\text {external }}\right)$. In model variants in which the weights varied not only across participants but also across experiments or postures, independent population distributions were estimated for each of these variables. The population distributions were Gaussians, for which mean and standard deviation were estimated during model fitting. In the binomial models, these Gaussians had $\mathcal{N}(1,2)$ priors for the mean values and Uniform $(0,2)$ priors for the standard deviations. In the logistic models, priors of the mean values were $\mathcal{N}(2,2)$ distributions. When the weights were held constant across participants we used an $\mathcal{N}(1,2)$ prior. Some model variants additionally estimated the task context parameters $\delta_{\text {anatomical }}$ and $\delta_{\text {external }}$. The free task context parameters (STL compared to TOJ and FTL compared to TOJ) had an $\mathcal{N}(1,2)$ prior. The logistic model variants additionally contained a free PSS parameter which always had an $\mathcal{N}(0,2)$ prior.

Goodness of fit and model comparison For model evaluation, the posterior predictive distribution was sampled: In each iteration, a data point $(k$, number of right- hand responses) was generated based on the current parameter estimates.

To visualize the goodness of fit, the posterior predictive distribution was plotted together with the observed values. A formalized test of the goodness of fit was performed using posterior predictive checks (Gelman et al., 1996), which compare the lack of fit of the model for the observed data with the lack of fit of the model when fitted to random data generated by the same model. The $\chi^{2}$-statistic was used to quantify the respective lack of fit. The comparison of the two resulting $\chi^{2}$ values determined the Bayesian $p$ value of the model. As these two discrepancies are in the ideal case identical, a suitable model has a Bayesian $p$ value near 0.5 , whereas values close to 0 or close to 1 indicate that the chosen model is not appropriate. Additionally, we calculated the determination coefficient $R^{2}$ and the root mean squared deviance (RMSD) of all samples from the posterior predictive distribution compared to the experimentally observed data. Note that RMSD is estimated larger and $R^{2}$ smaller, when using the full posterior predictive distribution rather than the maximum a posteriori (MAP) estimate as is often done. For model comparison, the Bayesian predictive information criterion (BPIC) (Ando, 2007) was assessed with model complexity $p V$ measured as half the posterior variance of the model-level deviance (Gelman et al., 2004).

Model validation We used a leave-one-out approach to identify the predictive capabilities of the best binomial and logistic models. Using MCMC-sampling, we acquired posterior predictive distributions rather than point estimates for the prediction of missing values. First, participants performance in uncrossed postures was predicted based on their performance in crossed posture conditions, and vice versa. Second, participants' performance in one task was used to predict performance in the other task(s). Third, individual participants' performance was predicted based on the data from all other participants.

The quality of these predictions was compared to the fit of posterior predictive distributions generated by the respective unconstrained models (Models 9 and 11 for the binomial and logistic models, respectively), and to the predictions of a Bayesian full linear regression model with random intercepts (for the binomial model) and a Bayesian full probit regression model with random intercepts (for the logistic model), respectively. For each comparison, we calculated paired $t$ tests on participant level RMSD values.

Parameter analysis During model evaluation, the posterior distribution of each free parameter was approximated. We characterized these distributions by calculating their MAP estimates and the respective $95 \%$ credible intervals (CI). Weight parameters $\omega_{\text {anatomical }}$ and $\omega_{\text {external }}$ were interpreted as reliably influential if their CI did not contain zero. This is because a weight of 0 would indicate that the respective response code was not considered for the response at all. Task context parameters $\delta_{\text {anatomical }}$ and $\delta_{\text {external }}$ were deemed reliable if their CI did not include 1 . This is because, a task context parameter of 1 would denote identity of the weights with the reference weights, that is, with the weights of the TOJ task, indicating that the parameter did not express a difference between tasks. 
To further analyze the estimated weight parameters, we correlated the MAPs of the individual anatomical weights $\left(\omega_{\text {anatomical }}\right)$ and the individual external weights $\left(\omega_{\text {external }}\right)$, as well as and each of these weight parameters with the participants' average crossing effects (that is, accuracy in uncrossed minus accuracy in crossed conditions).

\section{Results}

\section{Behavioral experiments}

Each participant performed three tactile localization tasks (TOJ, FTL, and STL, see Fig. 1), with uncrossed and crossed hands. A repeated measures ANOVA on accuracy data revealed a main effect of experiment $(F(1,18)=$ 113.25, $p<0.001, \eta^{2}=0.50$ ), a main effect of posture $\left(F(1,18)=80.68, p<0.001, \eta^{2}=0.57\right)$, and an interaction between the two factors $(F(1,18)=65.05, p<$ $0.001, \eta^{2}=0.32$; Fig. 4).

In the first experiment, TOJ, we observed a crossing effect (paired $t$ tests, accuracy: $t(18)=9.40, p<$ $0.001, d=2.16$, probit slope: $t(18)=12.53, p<$ $0.001, d=2.88$ ), demonstrating lower performance, that is, less button presses with the hand stimulated first, in crossed as compared to uncrossed postures.

In the second experiment, FTL, a crossing effect emerged as well (paired $t$ tests, accuracy: $t(18)=8.17, p<$ $0.001, d=1.87$ ), probit slope: $t(18)=11.59, p<$ $0.001, d=2.70$ ), but this crossing effect was smaller than the crossing effect in the TOJ task (paired $t$ tests of crossing effect difference scores, accuracy: $t(18)=$ $4.44, p<0.001, d=1.02$, probit slope: $t(18)=2.53, p=$ $0.021, d=0.60$ ).

In the third experiment, STL, a crossing effect (paired t-test $t(18)=3.75, p=0.001, d=0.86$ ) was evident as well. The STL crossing effect was smaller than the FTL

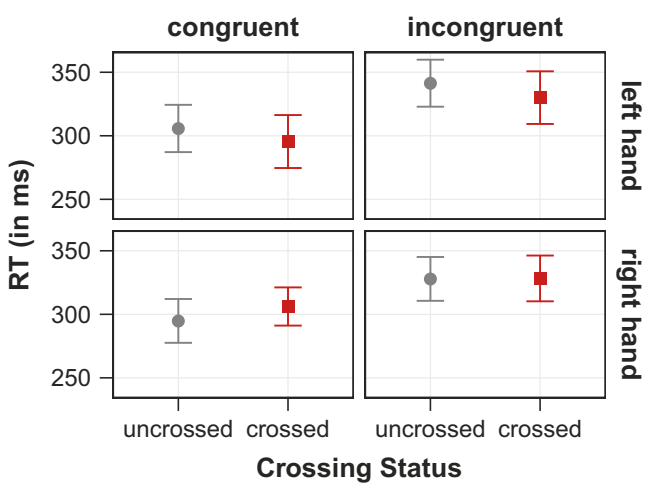

Fig. 5 Absence of a crossing effect in touch detection. Mean reaction times in the touch detection task are shown for uncrossed (grey) and crossed (red) postures. In congruent conditions a touch at the designated response hand had to be detected. In incongruent conditions the touch was applied to the other hand. Error bars show standard errors of the mean

crossing effect (paired $t$ test of crossing effect difference scores, accuracy: $t(18)=8.02, p<0.001, d=1.84)$ and the TOJ crossing effect (paired $t$ tests of crossing effect difference scores, accuracy: $t(18)=9.15, p<0.001, d=$ 2.10).

Pairwise correlations of the crossing effects from the three localization tasks revealed that they were positively correlated $\left(r_{\text {STL-FTL }}=0.57, p=0.011 ; r_{\text {STL-TOJ }}=\right.$ $0.46, p=0.047 ; r_{\text {FTL-TOJ }}=0.57, p<0.001$ ).

In the tactile detection task, an effect of stimulusresponse-congruency (repeated measures ANOVA, $\left.F(1,18)=27.38, p<0.001, \eta^{2}=0.04\right)$ but no crossing effect (repeated measures ANOVA, $F(1,18)=0.21, p=0.654, \eta^{2}<0.01$; post-hoc t-test of equivalence (Wellek, 2003), $p<0.050$, power $=0.42$ ) was observed in RT (Fig. 5).

In sum, crossing the hands resulted in performance impairments in all tactile localization tasks, but not in the touch detection task. Moreover, the size of the crossing

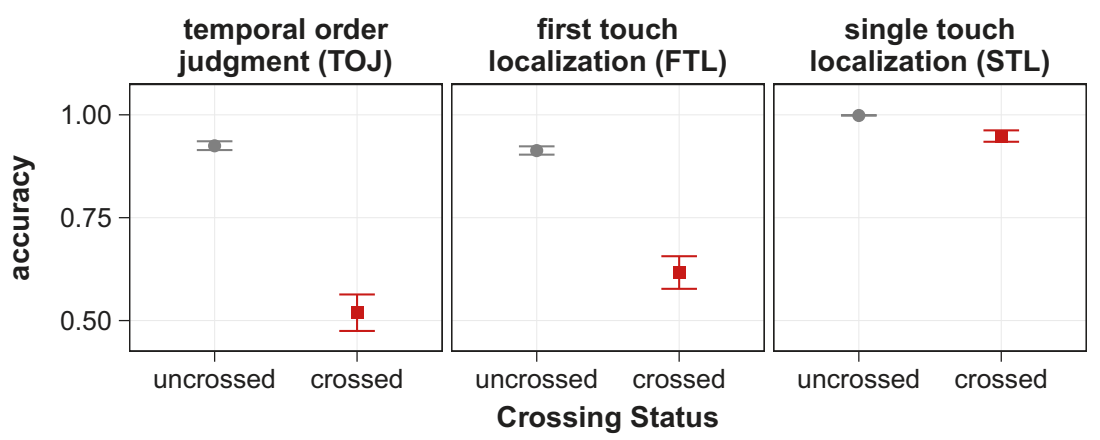

Fig. 4 Modulation of crossing effects across three different touch localization tasks. Performance in TOJ, FTL, and STL is shown as mean accuracy for uncrossed (grey) and crossed (red) postures. Error bars show standard errors of the mean. The size of the crossing effects, that is, the difference in performance between crossed and uncrossed postures, varied among experiments, demonstrating bottom-up as well as top-down influences on tactile localization 
effect was modulated by the number of stimuli and by the task instructions.

\section{Model}

Goodness of fit and model comparison We report goodness of fit for all models, quantified by the Bayesian $p$ value, the root mean square deviation (RMSD), and $R^{2}$, in Table 1. Model comparison was conducted based on Bayesian predictive information criterion (BPIC) values (Ando, 2007), which relate the model's deviance to its complexity (see Table 1).

SOA-independent binomial models provided, in general, a very good fit of participants' response patterns, indicated by Bayesian $p$ values around the ideal value of 0.5 and $R^{2}$ values higher than $90 \%$. Among the binomial models, the lowest BPIC value was obtained for a variant of the integration model, the pure conflict model (Model 3 see Fig. 6 for model fit). In this model, all free parameters were independent of posture. Individual anatomical and external weights were estimated for each participant, but these weights were constant across experiments. Integration model variants that comprised more free parameters (Models 1 and 2) than Model 3 obtained reliably higher BPIC values. This indicates that their only slightly better fit was compromised by their higher complexity. Variants of the integration model with fewer parameters (Models 4-6) than the pure conflict model obtained a worse fit and higher BPIC values. Model 6, the model with the smallest number of parameters and enforced constant weight parameters, even failed to converge with several sets of priors.

Variants of the non-integration model that had a higher number of free parameters than the pure conflict model, that is, model variants which comprised independent individual parameters for the three experiments (Models 9 and 10), obtained reliably higher BPIC values as well. Remarkably, the non-integration model with the same number of parameters as the pure conflict model obtained the highest BPIC value of all binomial models (Model 11). The drop in goodness of fit from Model 10 to Model 11 indicates that the non-integration model was less capable of simultaneously accounting for single participants performance across all three tasks than the pure conflict model.

For the logistic models posterior predictive checks consistently revealed Bayesian $p$ values lower than the optimal value of 0.5 . This is because there were data points in single participants' data that clearly deviated from an ideal psychophysical sigmoid response curve, especially in the crossed conditions (see Fig. 7, red dots). Accordingly, RMSD and $R^{2}$ measures were lower than those of the binomial models as well. Furthermore, the deviation of individual participants' responses from sigmoid curves was as well reflected in a higher deviance that, in turn, resulted in higher BPIC values. As a consequence, model complexity influenced the BPIC value to a lower degree than it had for the binomial models. The lowest BPIC value was obtained by the unconstrained model (Model 12), followed by the integration model with individually estimated PSS values (Model 7). Importantly, the integration model had a smaller BPIC value than the non-integration model with same number of free parameters (Model 13).

Model validation In a next step, we used a leave-one-out approach to examine how well the best binomial integration model, the pure conflict model (Model 3) and its logistic analogue (Model 8) predicted individual performance. First, crossed performance was predicted from uncrossed performance and vice versa. Second, performance in one of the tasks was used to predict performance in the other tasks, and third, single participants' response pattern was predicted based on the performance of the remaining participants. Prediction quality was compared to the predictions made by the unconstrained binomial and logistic models, and to the predictions made by a full Bayesian LMM with individual intercepts (for the binomial models), and a full Bayesian probit regression with individual intercepts (for the logistic models), respectively. All tests confirmed that predictions made by the pure conflict model and its logistic analogue achieved significantly smaller RMSD values than the predictions by the unconstrained models and by the statistical models (see Table 2). Predictions were most accurate when uncrossed performance was predicted from crossed performance. Predictions were less precise when crossed performance was predicted from uncrossed performance. When performance in one task was used to predict performance in the other tasks the pure conflict model had the highest advantage over the other tested models. Entire individual performance patterns were predicted with the lowest accuracy. Still, the predictions of the pure conflict model were reliably more accurate than predictions by the other models.

Parameter analysis In a last step, we specified the parameter estimates of the winner model, that is, of the pure conflict model (Model 3).

Figure 8 depicts the posterior distributions of the anatomical and the external task context parameters $\left(\delta_{\text {anatomical }}\right.$ and $\left.\delta_{\text {external }}\right)$. These parameters adjusted the individual anatomical and external weights across all participants for each experiment to capture general performance differences between the three tasks. The task context parameters were set to 1 in the TOJ task to provide a reference point for the other two tasks. Accordingly, a task context parameter estimate indicated a reliable experimentally induced weight difference in comparison to the TOJ task if its CI did not include 1 (a context parameter of 1 would indicate identical 


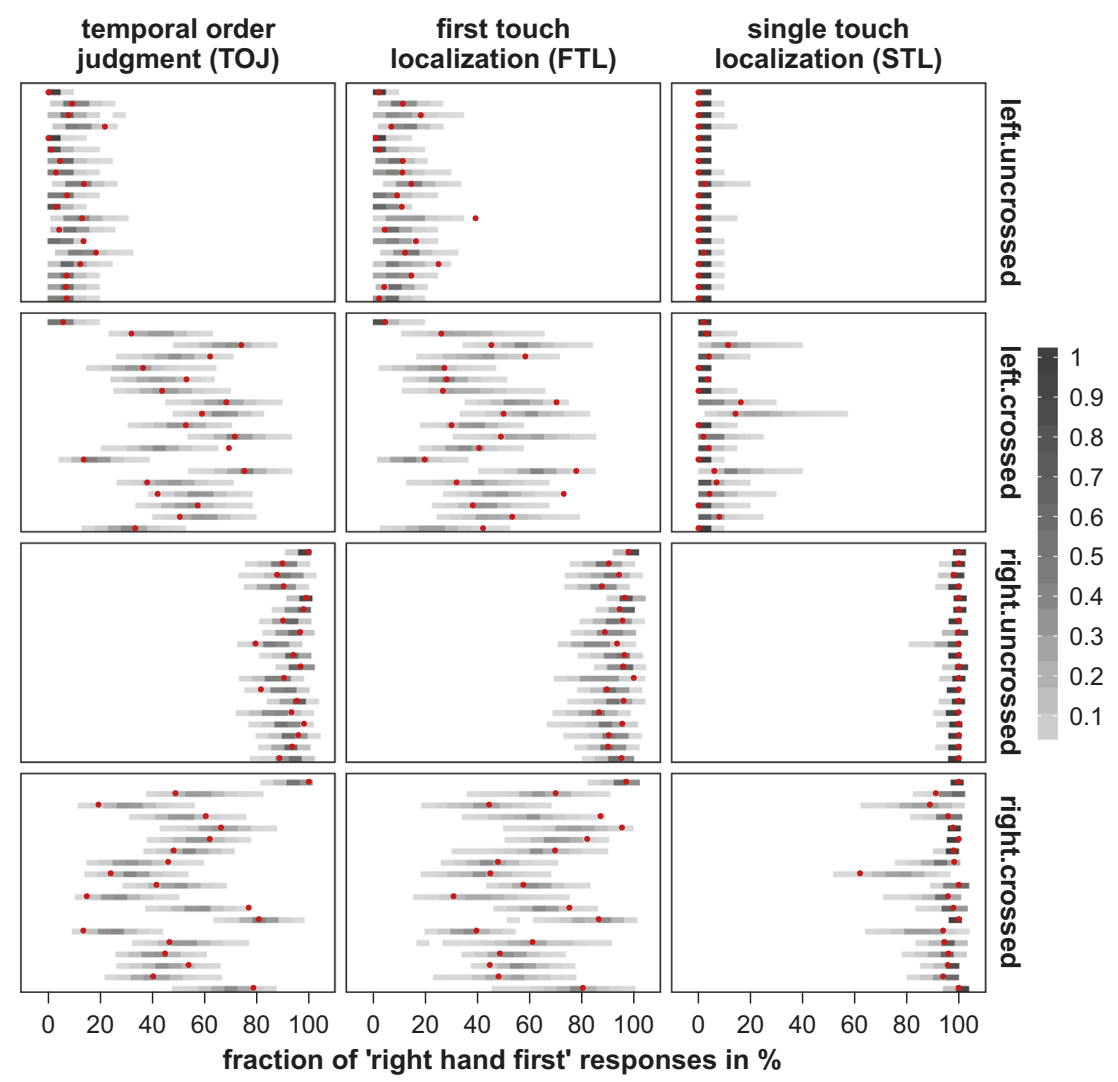

Fig. 6 Goodness of fit of the best model's (pure conflict model, Model 3) predictions for all three localization experiments. Posterior predictive distributions (grey bars), that is, frequency distributions of the fraction of "right-hand" responses as predicted by the model, are

weighing as in the TOJ task). In the STL task, the anatomical task context parameter exceeded 1 , indicating that all participants weighed the anatomical coordinates in the STL task more strongly than in the TOJ task $\left(\delta_{\text {anatomical }}(\mathrm{STL})\right.$ : MAP $=4.02,95 \% \mathrm{CI}=[3.66,4.44])$. In contrast, no difference between TOJ and STL tasks was evident in the external task context parameter $\left(\delta_{\text {external }}(\mathrm{STL}): \mathrm{MAP}=1.02,95 \%\right.$ $\mathrm{CI}=[0.68,1.12])$. In the FTL task, the anatomical task context parameter was slightly larger than that of the TOJ task $\left(\delta_{\text {anatomical }}(\mathrm{FTL}): \mathrm{MAP}=1.11,95 \% \mathrm{CI}=[1.04,1.18]\right)$. In contrast, the external task context parameter indicated a generally lower external weight in the FTL than in the TOJ task $\left(\delta_{\text {external }}(\mathrm{FTL}): \mathrm{MAP}=0.75,95 \% \mathrm{CI}=[0.71,0.82]\right)$.

The posterior distributions of individual weight parameters ( $\omega_{\text {anatomical }}$ and $\left.\omega_{\text {external }}\right)$ are shown in Fig. 9.

We assessed the correlation of the MAPs of the individual anatomical and external weights, as well as of each weight MAP with participants average crossing effect in accuracy (see Fig. 10). There was no significant correlation between anatomical and external weights $(\mathrm{t}(17)=-0.92, \mathrm{p}=0.370$, $r=-0.22)$. However, anatomical weights correlated negatively $(\mathrm{t}(17)=-4.33, \mathrm{p}<0.001, r=-0.72)$ and external weights correlated positively $(\mathrm{t}(17)=5.05, \mathrm{p}<0.001, r=$ plotted with the observed fractions (red dots). Data from three tactile localization tasks is shown separately for each hand posture and stimulated hand. Each line represents one participant

0.77) with the size of the crossing effect. When anatomical and external weights are transformed into an unbounded gain and a bounded weight parameter, the interindividual differences in the size of the crossing effect are fully captured by the weighting of the anatomical and external response codes (see Supplementary Material).

\section{Discussion}

The aim of the present study was to characterize the processing principles that underlie tactile localization responses. In particular, tactile localization performance might either be determined by the ability to transform an anatomical into an external response code, or, instead, by the integration of anatomically and externally mapped response codes. To address this issue, we designed probabilistic models based on these two alternative hypotheses, and tested their ability to account for touch location choices of human participants. Participants completed three different tactile localization tasks and one touch detection task. In all of these tasks a conflict between left-right mappings in anatomical and external reference frames was created through hand 


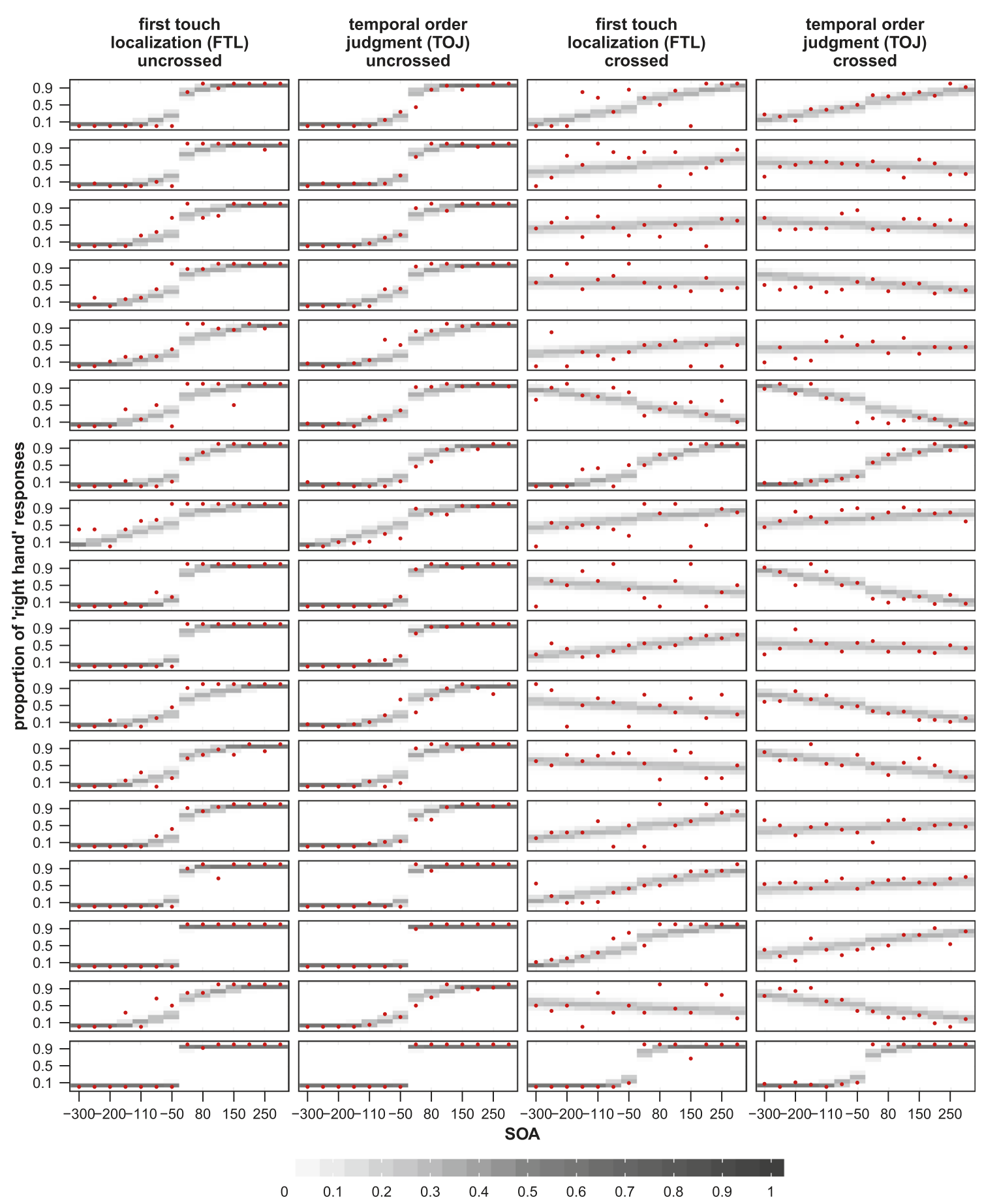

Fig. 7 Goodness of fit of the logistic analogue of the pure conflict model's predictions for psychophysical functions from TOJ and FTL tasks. Posterior predictive distributions (vertical grey bars) are plotted with the observed response ratios (red dots). The ratio of "right-hand" responses in the FTL and TOJ localization tasks is shown as function of SOA (depicted on a non-linear scale). Each row shows performance of one participant in both tasks and in

crossing. Crossing effects, that is, lower performance in the crossed as compared to the uncrossed posture conditions, emerged in all three localization tasks. These crossing effects correlated across experiments, suggesting that a crossed as well as uncrossed postures. Some participants show inverted response patterns in the crossed posture. This type of response pattern has been reported previously (Yamamoto \& Kitazawa, 2001a; Wada et al., 2004) and the curve has been fitted with a dedicated function (Yamamoto \& Kitazawa, 2001a). In contrast, our model accounted for these inverted response patterns without specialized adjustments

common mechanism underlies tactile localization responses in all three experiments. The integration model outperformed the model based on external response codes alone. The best integration model fitted individual differences in 


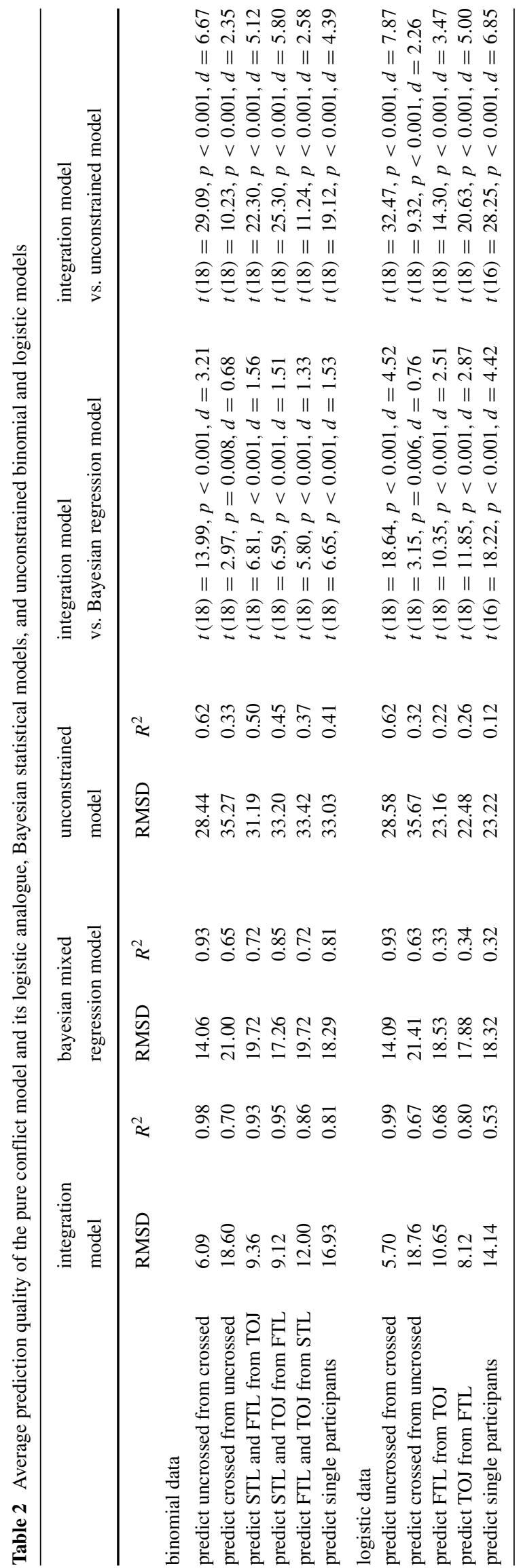

localization performance between tasks across participants and used identical reference frame weights in both crossed and uncrossed postures. Thus, this model was based on the assumption that the external response code, that is, the outcome of the genuine tactile remapping process was unaffected by posture. Instead, crossing effects were exclusively accounted for by integration of conflicting response codes.

Integration of response codes rather than reliability of the external response mapping determines touch location choices Based on our model comparisons, we suggest that crossing effects reflect the brain's general strategy to integrate all available pieces of information characterizing an event, for example, in the context of choosing tactile stimulus location. This strategy is adequate in most everyday situations. When the hands are uncrossed, anatomical and external reference frames provide redundant information and, therefore, the external reference frame seems to support tactile processing (Ruzzoli \& Soto-Faraco, 2014). However, in the crossed posture left-right mappings are incongruent between anatomical and external reference frames, and integration of the externally coded response hinders performance in anatomical touch localization tasks. Crossing effects were best explained by the integration model. This model assumes that the probability of each localization response is based on the integration of the independently weighted anatomical and external left-right response codes of the touch. Moreover, a single weight parameter for each reference frame was sufficient to account for uncrossed and for crossed performance, indicating that the integration follows the same principles in both postures.

Effects of body posture on touch processing have often been attributed to the external reference frame of touch (Driver \& Spence, 1998; Aglioti et al., 1999; Kennett et al., 2001; Yamamoto \& Kitazawa, 2001a; Shore et al., 2002; Soto-Faraco et al., 2004; Röder et al., 2004; Eimer et al., 2004; Bolognini \& Maravita, 2007; Azañón \& Soto-Faraco, 2008; Heed et al., 2012; Buchholz et al., 2011; 2013). This is because body posture determines the location of the stimulated skin region in space, but should not influence the processing of any other tactile stimulus characteristics (Forster \& Eimer, 2004). Consequently, crossing effects have been suggested to arise from error-prone, unreliable external left-right response mappings in crossed compared to uncrossed hand postures (Yamamoto \& Kitazawa, 2001a; Azañón \& Soto-Faraco, 2007; Soto-Faraco \& Azañón, 2013). In this framework, the existence of crossing effects suggests that responses, for example, in the TOJ task, are derived based on the external representation of touch alone (Kitazawa, 2002). In the present study, the model that based the choice between "left" and "right" on external response codes alone and, thus, accounted for crossing effects by reduced reliability of the external response codes in the 


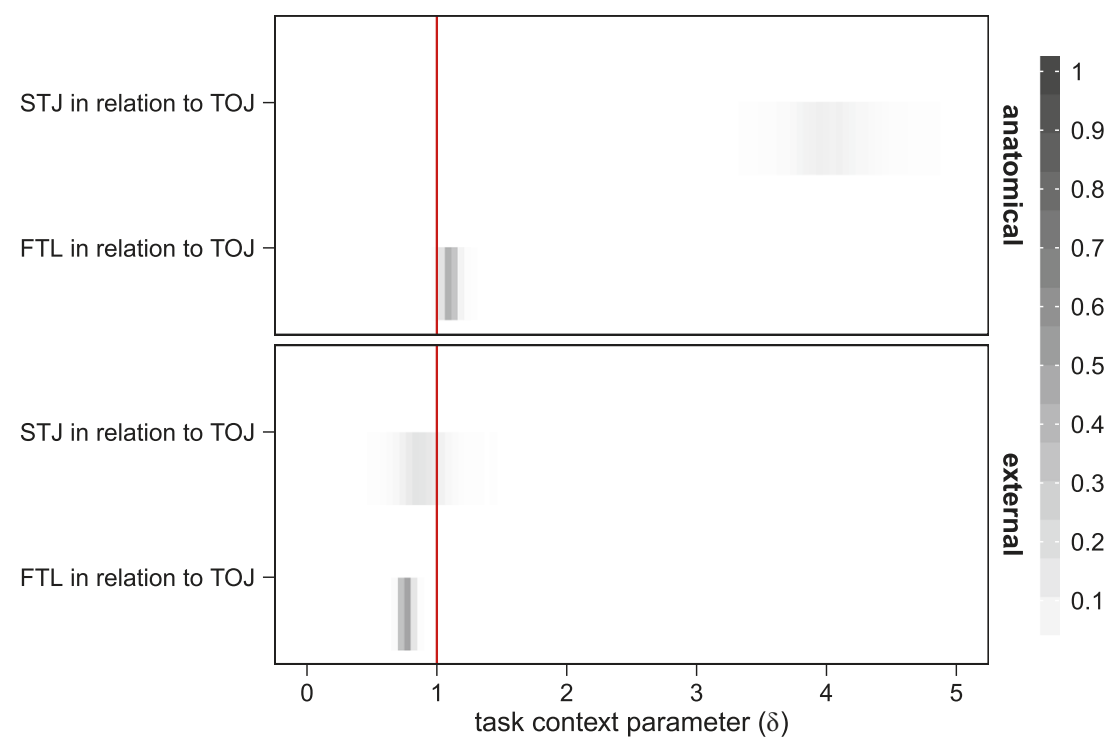

Fig. 8 Posterior distributions of the task context parameters. The estimates are derived from the pure conflict model (Model 3), which used identical participant-specific weights across experiments and postures.
The task context parameter was fixed to 1 for the TOJ task, therefore, a reliable task context effect for the other experiments should differ from 1 crossed postures had a considerably worse fit than the integration model, discounting problems in the derivation of the external left-right mappings in the crossed posture as a source of crossing effects.

Furthermore alternative explanations of crossing effects that refer to the unfamiliarity of the crossed posture (Azañon et al., 2010b; Longo et al., 2010) or the need for interhemispheric transmission in the crossed posture (Buchholz et al., 2012) seem improbable, given the lack of an influence of hand crossing on touch detection. Further, these alternative explanations do not receive support from our modeling results; our results suggest that crossing effects are not due to posture per se, but, instead, to the anatomical and external response codes being incongruent in crossed, but not in uncrossed postures.

Weighted integration accounts for previous findings The weighted integration account suggests a new view on previous results. We will point out some examples of how weighted integration of anatomical and external localization responses can provide an alternative interpretation of existing findings.

Integration of information coded in different reference frames appears to depend strongly on (visual) experience

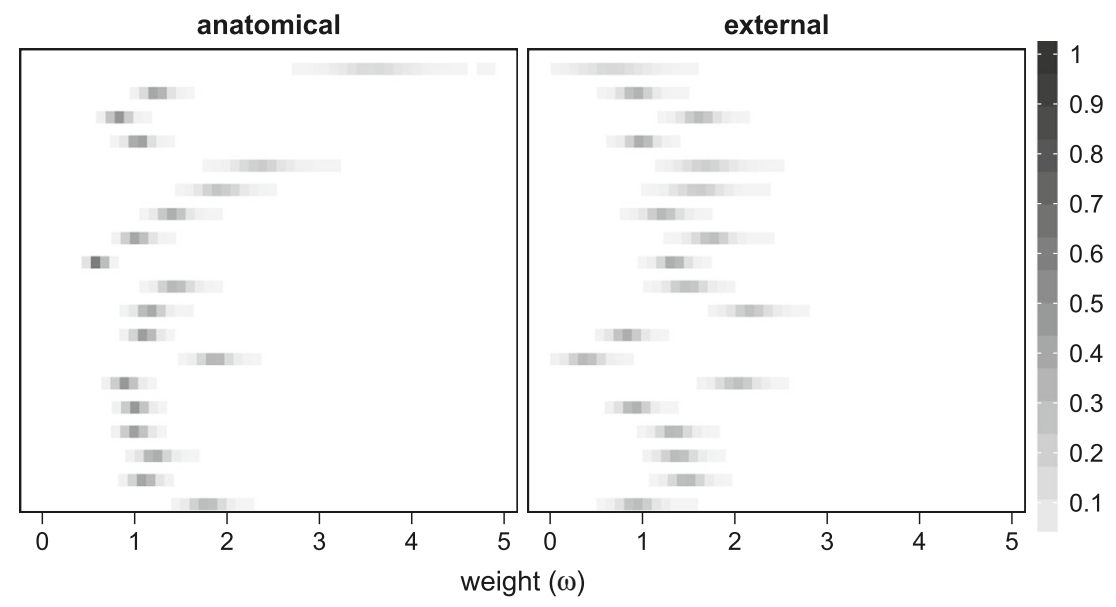

Fig. 9 Single participants' weight estimates. Posterior distributions of the individual weights (grey bars, each line represents one participant order of participants is equal to participants order in Fig. (6)) are shown. The weights derive from the best integration model (Model 3, pure conflict model), which used identical weights across experiments and postures. No upper bounded was set for the weights; posterior distributions of bounded weights are presented in the Supplementary Material 


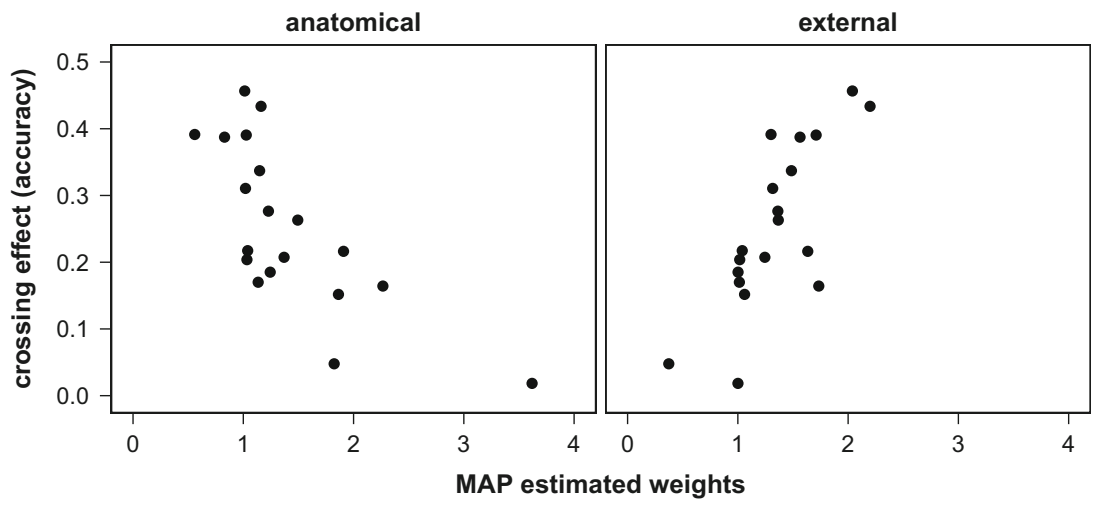

Fig. 10 Relation between crossing effects and weight estimates. MAP estimates of the individual anatomical and external weights $\left(\omega_{\text {anatomical }}\right.$ and $\left.\omega_{\text {external }}\right)$ plotted against the respective participant's crossing effect in accuracy averaged across experiments

during development (Röder et al., 2004). Children under the age of 5.5 years did not show crossing effects in tactile TOJ (Pagel et al., 2009), although external tactile coordinates seem to be available at the age of 10 months (Bremner et al., 2008). This implies that younger children may not yet integrate anatomical and external information at a younger age, although both types of information are available to them (Röder et al., 2013, 2014).

Crossing effects in tactile TOJ occur as well when the tactile stimuli are applied to crossed and uncrossed drumsticks held in the uncrossed hands (Yamamoto \& Kitazawa, 2001b). When both the hands and the sticks were crossed such that the tips of the sticks each lied in their regular hemispace, TOJ accuracy recovered. Similarly, TOJ performance recovered for tactile stimuli applied to the little fingers when the hands were crossed, but the little fingers were additionally crossed back into their regular hemifield (Heed et al., 2012). This compensation of the crossing effect by double-crossing seems counter-intuitive, but is predicted by our model: in the described situations that involved two crossings, a touch on left hand is located in the left hemispace, so that no conflict arises between anatomical and external left-right mappings. Likewise, crossing the fingers improved touch localization when the number of left-right conflicts for anatomical and external touch representations at fingers and hands was reduced in crossed compared to uncrossed postures (Badde et al., 2014c).

Touch processing has been reported to depend on concurrent visual information. Some studies have indicated that concurrent non-informative vision leads to a re-weighting of spatial information in favor of the anatomical reference frame (Lloyd et al., 2003; Rigato et al., 2013), whereas others have suggested that the external reference frame is weighted stronger (Newport et al., 2002). Although the picture arising from these studies does not yet appear consistent, it clearly supports our notion that reference frame weighing is a key determinant of tactile spatial processing.
Psychological interpretation of weight parameters If integration of information from different reference frames is indeed central to tactile processing, then it is of interest to relate the specific weights to psychological processes and to brain function. The integration model posits that inter- and intraindividual differences in the size of crossing effects are due to varying degrees of conflict. In turn, the degree of conflict is determined by the weights assigned to anatomical and external reference frames. On a physiological level, a higher weight might be associated with higher neuronal activity. In turn, higher neuronal activity may account for higher accuracy and shorter response times, a principle well-known from diffusion models (Philiastides et al., 2006). In popular neurophysiological models of multisensory spatial transformation (Ma et al., 2006; Deneve et al., 2001) the reference frame weights might relate to the gain of the probabilistic population codes of unimodal neurons. On the psychological level, the weights assigned to the two reference frames may be interpreted as measures of the relevance assigned to them in the integration process. In our experiments the weights correlated with the size of the crossing effect: the lower the anatomical weights and the higher the external weights were, the larger were the crossing effects in our tasks. In addition, crossing effects were correlated across experiments. The pure integration model accounted for intra-individual differences in crossing effects between experiments with adjustments of a parameter that is common to all participants. Hence, participants seem to have an individual default integration schema which they adjust to fit the current demands in a common way.

Our experimental data indicate that a reference frame's importance depends on bottom-up factors like stimulus properties, as well as on top-down influences, such as task instructions. Other bottom-up influences such as the reliability of sensory (Ernst \& Banks, 2002) or sensimotor (Körding \& Wolpert, 2004) information, or target modality (Sober \& Sabes, 2005) may influence our model's weights, 
too, but were not within the scope of the current study. Moreover, the present parameter estimates invite the speculation that bottom-up influences, such as the number of stimuli, are reflected in the anatomical weights, whereas top-down influences, such as task instructions, bear on the external weights: Indeed, the anatomical representation of touch is associated with the primary somatosensory cortices, that store all sensory characteristics of a touch. In contrast, the external representation of touch is presumably established later and in higher-order brain regions (Azañón \& Soto-Faraco, 2008; Heed \& Röder, 2010; Overvliet et al., 2011; Soto-Faraco \& Azañón, 2013; Rigato et al., 2013). The latter are tightly connected to frontal brain areas associated with cognitive control processes.

\section{Touch localization in external space is automatic and} top-down controlled Our behavioral results imply an apparent contradiction. In the present study, task instructions were phrased in anatomical terms (that is, participants were always asked to respond with the stimulated hand, and the side of space was not mentioned). Yet, as in previous studies, the external reference frame of touch had a remarkable influence on tactile task performance, supporting the view that the remapping of touch into external space is an automatic process (Kitazawa, 2002; Röder et al., 2004; Azañón et al., 2010a). However, a small variation of the task instructions (TOJ vs. FTL) altered the size of the crossing effect in spite of identical physical stimulation and required processing steps. This modulation, independent of its direction, is incompatible with the notion of automaticity. In line with this finding, TOJ crossing effects have been shown to be modulated by processing load (Badde et al., 2014) as well as by the task instructions in a second, independent task (Badde et al., 2015). To reconcile these results, we have previously suggested (Badde et al., 2014; Badde et al., 2015) that the first step of tactile localization, stimulus remapping, proceeds automatically. In contrast, the second step of tactile localization, integration of anatomically and externally mapped information, is subject to top-down control. Accordingly, in the framework of our proposed model, task instructions, modeled as task context parameter, affected the integration of response codes implied by anatomical and external reference frames.

\section{Conclusions}

In sum, we have demonstrated that tactile spatial processing is subject to both bottom-up and top-down factors. Integration of conflicting information can account for errors in tactile localization tasks that arise when anatomical and external mappings of a touch are incongruent. The integration model we proposed captures individual differences in tactile localization tasks performance based on the weights assigned to anatomical and external mappings, but characterizes differences between experiments with parameters common to all participants. This feature suggests common processing principles across individuals. With this account, it is obsolete to posit an impairment of the genuine tactile remapping process, that is, the transformation of anatomical information into external space. Instead our model suggests that touch location responses are established by one unified mechanism, irrespective of posture.

Acknowledgements SB was supported by the German-Sino Research Training Group CINACS, DFG GRK 1247/1 and 1247/2. TH is supported by an Emmy Noether grant from the German Research Foundation (DFG), He 6368/1-1. We thank T. Thöring for help with data acquisition.

\section{References}

Aglioti, S., Smania, N., \& Peru, A. (1999). Frames of reference for mapping tactile stimuli in brain-damaged patients. Journal of Cognitive Neuroscience, 11(1), 67-79.

Ando, T. (2007). Bayesian predictive information criterion for the evaluation of hierarchical Bayesian and empirical Bayes models. Biometrika, 94(2), 443-458. doi:10.1093/biomet/asm017

Azañón, E., \& Soto-Faraco, S. (2007). Alleviating the 'crossed-hands' deficit by seeing uncrossed rubber hands. Experimental Brain Research, 182(4), 537-548. doi:10.1007/s00221-007-1011-3

Azañón, E., \& Soto-Faraco, S. (2008). Changing reference frames during the encoding of tactile events. Current Biology, 18(14), 1044 1049. doi:10.1016/j.cub.2008.06.045

Azañón, E., Camacho, K., \& Soto-Faraco, S. (2010a). Tactile remapping beyond space. European Journal of Neuroscience, 31(10), 1858-1867. doi:10.1111/j.1460-9568.2010.07233.x

Azañón, E., Longo, M.R., Soto-Faraco, S., \& Haggard, P. (2010b). The posterior parietal cortex remaps touch into external space. Current Biology, 20(14), 1304-1309.

Azañón, E., Stenner, M.P., Cardini, F., \& Haggard, P. (2015). Dynamic tuning of tactile localization to body posture. Current Biology. doi:10.1016/j.cub.2014.12.038

Badde, S., Heed, T., \& Röder, B. (2013). Modelling body posture effects on reference frame integration. In Abstract 14th International Multisensory Research Forum, Jerusalem, Israel (May 3-6, 2013).

Badde, S., Heed, T., \& Röder, B. (2014). Processing load impairs coordinate integration for the localization of touch. Attention, Perception, \& Psychophysics, 76(4), 1136-1150. doi:10.3758/s13414-013-0590-2

Badde, S., Röder, B., \& Heed, T. (2015). Flexibly weighted integration of tactile reference frames. Neuropsychologia, $70,367-374$.

Badde, S., Röder, B., \& Heed, T. (2014c). Multiple spatial representations determine touch localization on the fingers. Journal of Experimental Psychology: Human Perception and Performance, 40(2), 784-801. doi:10.1037/a0034690

Bolognini, N., \& Maravita, A. (2007). Proprioceptive alignment of visual and somatosensory maps in the posterior parietal cortex. Current Biology, 17(21), 1890-1895. doi:10.1016/j.cub.2007.09.057 
Bremner, A.J., Holmes, N.P., \& Spence, C. (2008). Infants lost in (peripersonal) space? Trends in Cognitive Sciences, 12(8), 298305. doi:10.1016/j.tics.2008.05.003

Buchholz, V.N., Jensen, O., \& Medendorp, W.P. (2011). Multiple reference frames in cortical oscillatory activity during tactile remapping for saccades. Journal of Neuroscience, 31(6), 864-16,871. doi:10.1523/JNEUROSCI.3404-11.2011

Buchholz, V.N., Goonetilleke, S.C., Medendorp, W.P., \& Corneil, B.D. (2012). Greater benefits of multisensory integration during complex sensorimotor transformations. Journal of Neurophysiology, 107(11), 3135-3143. doi:10.1152/jn.01188.2011

Buchholz, V.N., Jensen, O., \& Medendorp, W.P. (2013). Parietal oscillations code nonvisual reach targets relative to gaze and body. Journal of Neuroscience, 33(8), 3492-3499. doi:10.1523/ JNEUROSCI.3208-12.2013.

Cadieux, M.L., Barnett-Cowan, M., \& Shore, D.I. (2010). Crossing the hands is more confusing for females than males. Exp Brain Res, 204(3), 431-446. doi:10.1007/s00221-010-2268-5

Canzoneri, E., Ferrè E.R, \& Haggard P. (2014). Combining proprioception and touch to compute spatial information. Experimental Brain Research, 232(4), 1259-1266. doi:10.1007/s00221-014-3842-z

Deneve, S., Latham, P.E., \& Pouget, A. (2001). Efficient computation and cue integration with noisy population codes. Nature Neuroscience, 4(8), 826-831. doi:10.1038/90541

Driver, J., \& Spence, C. (1998). Cross-modal links in spatial attention. Philosophical Transactions of the Royal Society B: Biological Sciences, 353(1373), 1319-1331. doi:10.1098/rstb.1998.0286

Eimer, M., Forster, B., Fieger, A., \& Harbich, S. (2004). Effects of hand posture on preparatory control processes and sensory modulations in tactile-spatial attention. Clinical Neurophysiology, 115(3), 596-608. doi:10.1016/j.clinph.2003.10.015

Ernst, M.O., \& Banks, M.S. (2002). Humans integrate visual and haptic information in a statistically optimal fashion. Nature, 415(6870), 429-433. doi:10.1038/415429a

Finney, D. (1947). Probit analysis: a statistical treatment of the sigmoid response curve, 1st edn. Cambridge: Cambridge University Press.

Forster, B., \& Eimer, M. (2004). The attentional selection of spatial and non-spatial attributes in touch: Erp evidence for parallel and independent processes. Biological Psychology, 66(1), 1-20. doi:10.1016/j.biopsycho.2003.08.001

Gelman, A., Meng, X.L., \& Stern, H. (1996). Posterior predictive assessment of model fitness via realized discrepancies. Statistica Sinica, 6(4), 733-760.

Gelman, A., Carlin, J.B., Stern, H.S., \& Rubin, D.B. (2004). Bayesian data analysis. Chapman \& Hall, Boca Raton, FL, USA.

Haggard, P., Taylor-Clarke, M., \& Kennett, S. (2003). Tactile perception, cortical representation and the bodily self. Current Biology, 13(5), R170-R173.

Heed, T., \& Azañón, E. (2014). Using time to investigate space: a review of tactile temporal order judgments as a window onto spatial processing in touch. Front Psychology, 5. doi:10.3389/fpsyg. 2014.00076

Heed, T., \& Röder, B. (2010). Common anatomical and external coding for hands and feet in tactile attention: evidence from eventrelated potentials. Journal of Cognitive Neurosciences, 22(1), 184-202. doi:10.1162/jocn.2008.21168

Heed, T., Backhaus, J., \& Röder, B. (2012). Integration of hand and finger location in external spatial coordinates for tactile localization. Journal of Experimental Psychology: Human Perception and Performance, 38(2), 386-401. doi:10.1037/a0024059

Heed, T., Buchholz, V.N., Engel, A.K., \& Röder, B. (2015). Tactile remapping: from coordinate transformation to integration in sensorimotor processing. Trends in Cognitive
Sciences, 19(5), 251-258. doi:10.1016/j.tics.2015.03.001. http:// www.sciencedirect.com/science/article/pii/S1364661315000492

Holm, S. (1979). A simple sequentially rejective multiple test procedure. Scandinavian Journal of Statistics, 6(2), 6570.

Kóbor, I., Füredi, L., Kovács, G., Spence, C., \& Vidnyánszky, Z. (2006). Back-to-front: improved tactile discrimination performance in the space you cannot see. Neuroscience Letters, 400(1-2), 163-167. doi:10.1016/j.neulet.2006.02.037

Kennett, S., Eimer, M., Spence, C., \& Driver, J. (2001). Tactilevisual links in exogenous spatial attention under different postures: convergent evidence from psychophysics and ERPs. Journal of Cognitive Neuroscience, 13(4), 462-478.

Kitazawa, S. (2002). Where conscious sensation takes place. Consciousness and Cognition, 11(3), 475-477. doi:10.1016/S1053-8100(02)00031-4

Kitazawa, S., Moizumi, S., Okuzumi, A., Saito, F., Shibuya, S., Takahashi, T., Wada, M., \& Yamamoto, S. (2008). Reversal of subjective temporal order due to sensory and motor integrations. In Haggard, P., Rossetti Y, \& Kawato M (Eds.) Sensorimotor foundations of higher cognition, Attention and Performance, Oxford University Press, New York, USA, chap Reversal of subjective temporal order due to sensory and motor integrations (pp. 73-97): Oxford University Press.

Körding, K.P., \& Wolpert, D.M. (2004). Bayesian integration in sensorimotor learning. Nature, 427(6971), 244-247. doi:10.1038/nature02169

Lloyd, D.M., Shore, D.I., Spence, C., \& Calvert, G.A. (2003). Multisensory representation of limb position in human premotor cortex. Nature Neuroscience, 6(1), 17-18. doi:10.1038/nn991

Longo, M.R., Azañón, E., \& Haggard, P. (2010). More than skin deep: body representation beyond primary somatosensory cortex. Neuropsychologia, 48(3), 655-668. doi:10.1016/j.neuropsychologia.2009.08.022

Lunn, D., Thomas, A., Best, N., \& Spiegelhalter, D. (2000). Winbugs - A Bayesian modelling framework: concepts, structure, and extensibility. Statistics and Computing, 10(4), 325337.

Ma, W.J., Beck, J.M., \& Pouget, A. (2006). Bayesian inference with probabilistic population codes. Nature Neurosciences, 9(11), 1432-1438. doi:10.1038/nn1790

Newport, R., Rabb, B., \& Jackson, S.R. (2002). Noninformative vision improves haptic spatial perception. Current Biology, 12(19), 1661-1664. doi:10.1016/S0960-9822(02)01178-8

Overvliet, K.E., Azañón, E., \& Soto-Faraco, S. (2011). Somatosensory saccades reveal the timing of tactile spatial remapping. Neuropsychologia, 49(11), 3046-3052. doi:10.1016/j.neuropsychologia.2011.07.005

Pagel, B., Heed, T., \& Röder, B. (2009). Change of reference frame for tactile localization during child development. Developmental Science, 12(6), 929-937. doi:10.1111/j.1467-7687.2009.00845.x

Penfield, W., \& Boldrey, E. (1937). Somatic motor and sensory representation in the cerebral cortex of man as studied by electrical stimulation. Brain, 60(4), 389-443. doi:10.1093/brain/60.4.389

Philiastides, M.G., Ratcliff, R., \& Sajda, P. (2006). Neural representation of task difficulty and decision making during perceptual categorization: a timing diagram. Journal Neuroscience, 26(35), 8965-8975. doi:10.1523/JNEUROSCI.1655-06.2006

Pouget, A., Ducom, J.C., Torri, J., \& Bavelier, D. (2002). Multisensory spatial representations in eye-centered coordinates for reaching. Cognition, 83(1), B1-11. doi:10.1016/S0010-0277(01)00163-9

Röder, B., Rösler, F., \& Spence, C. (2004). Early vision impairs tactile perception in the blind. Current Biology, 14(2), 121-124. doi:10.1016/S0960-9822(03)00984-9 
Röder, B., Pagel, B., \& Heed, T. (2013). The implicit use of spatial information develops later for crossmodal than for intramodal temporal processing. Cognition, 126(2), 301-306. doi:10.1016/j.cognition.2012.09.009

Röder, B., Heed, T., \& Badde, S. (2014). Development of the spatial coding of touch: ability vs. automaticity. Developmental Science, 17(6), 944-945. doi:10.1111/desc.12186

Renzi, C., Bruns, P., Heise, K.F., Zimerman, M., Feldheim, J.F., Hummel, F.C., \& Röder B (2013). Spatial remapping in the audiotactile ventriloquism effect: a TMS investigation on the role of the ventral intraparietal area. Journal of cognitive neuroscience, 25(5), 790-801.

Rigato, S., Bremner, A.J., Mason, L., Pickering, A., Davis, R., \& van Velzen, J. (2013). The electrophysiological time course of somatosensory spatial remapping: vision of the hands modulates effects of posture on somatosensory evoked potentials. European Journal Neuroscience, 38(6), 2884-2892. doi:10.1111/ejn.12292

Rouder, J.N., \& Lu, J. (2005). An introduction to Bayesian hierarchical models with an application in the theory of signal detection. Psychonomic Bulletin and Review, 12(4), 573604.

Ruzzoli, M., \& Soto-Faraco, S. (2014). Alpha stimulation of the human parietal cortex attunes tactile perception to external space. Current Biology, 24(3), 329-332. doi:10.1016/j.cub.2013.12.029

Schicke, T., \& Röder, B. (2006). Spatial remapping of touch: confusion of perceived stimulus order across hand and foot. Proceedings National Academie Science U S A, 813(31:11), 808-11. doi:10.1073/pnas.0601486103
Shore, D.I., Spry, E., \& Spence, C. (2002). Confusing the mind by crossing the hands. Cognitive Brain Research, 14(1), 153-163. doi:10.1016/S0926-6410(02)00070-8

Sober, S.J., \& Sabes, P.N. (2005). Flexible strategies for sensory integration during motor planning. Nature Neuroscience, 8(4), 490497. doi: $10.1038 / \mathrm{nn} 1427$

Soto-Faraco, S., \& Azañón, E. (2013). Electrophysiological correlates of tactile remapping. Neuropsychologia, 51(8), 1584-1594. doi:10.1016/j.neuropsychologia.2013.04.012

Soto-Faraco, S., Ronald, A., \& Spence, C. (2004). Tactile selective attention and body posture: assessing the multisensory contributions of vision and proprioception. Percept Psychophysics, 66(7), 1077-1094.

Spence, C., Pavani, F., Maravita, A., \& Holmes, N. (2004). Multisensory contributions to the 3-D representation of visuotactile peripersonal space in humans: evidence from the crossmodal congruency task. Journal of Physiology - Paris, 98(1-3), 171-189. doi:10.1016/j.jphysparis.2004.03.008

Wada, M., Yamamoto, S., \& Kitazawa, S. (2004). Effects of handedness on tactile temporal order judgment. Neuropsychologia, 42(14), 1887-1895. doi:10.1016/j.neuropsychologia.2004.05.009

Wellek, S. (2003). Testing statistical hypotheses of equivalence CRC Press, Boca Raton, FL, USA.

Yamamoto, S., \& Kitazawa, S. (2001a). Reversal of subjective temporal order due to arm crossing. Natural Neuroscience, 4(7), 759765. doi: $10.1038 / 89559$

Yamamoto, S., \& Kitazawa, S. (2001b). Sensation at the tips of invisible tools. Nature Neuroscience, 4(10), 979-980. doi:10.1038/nn721 